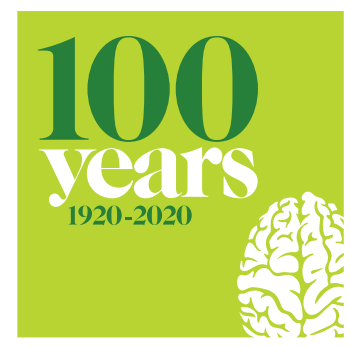

\title{
Original research: Second IVlg course in Guillain-Barré syndrome with poor prognosis: the non-randomised ISID study
}

\author{
Christine Verboon (D) , ${ }^{1}$ Bianca van den Berg, ${ }^{1}$ David R Cornblath, ${ }^{2}$ Esmee Venema, ${ }^{1,3}$ \\ Kenneth C Gorson, ${ }^{4}$ Michael P Lunn, ${ }^{5}$ Hester Lingsma, ${ }^{3}$ Peter Van den Bergh, ${ }^{6}$ \\ Thomas Harbo, ${ }^{7}$ Kathleen Bateman, ${ }^{8}$ Yann Pereon, ${ }^{9}$ Søren H Sindrup, ${ }^{10}$ \\ Susumu Kusunoki (D) ," James Miller, ${ }^{12}$ Zhahirul Islam, ${ }^{13}$ Hans-Peter Hartung, ${ }^{14}$ \\ Govindsinh Chavada, ${ }^{15}$ Bart C Jacobs, ${ }^{1,16}$ Richard A C Hughes, ${ }^{17}$ Pieter A van Doorn, ${ }^{1}$ \\ The IGOS Consortium
}

For numbered affiliations see end of article.

\section{Correspondence to} Prof Dr Pieter A van Doorn Neurology, Erasmus MC, 3000 CA, Rotterdam, The Netherlands; p.a.vandoorn@ erasmusmc.n

Received 25 June 2019 Revised 20 August 2019 Accepted 18 September 2019 Published Online First 5 October 2019

\section{SLinked}

- http://dx.doi.org/10.1136/ jnnp-2019-322361

\section{Check for updates}

(C) Author(s) (or their employer(s)) 2020. No commercial re-use. See rights and permissions. Published by BMJ.

To cite: Verboon $C$, van den Berg B, Cornblath DR, et al. J Neurol Neurosurg Psychiatry 2020;91:113-121.

\section{ABSTRACT}

Objective To compare disease course in patients with Guillain-Barré syndrome (GBS) with a poor prognosis who were treated with one or with two intravenous immunoglobulin (IVIg) courses.

Methods From the International GBS Outcome Study, we selected patients whose modified Erasmus GBS Outcome Score at week 1 predicted a poor prognosis. We compared those treated with one IVIg course to those treated with two IVIg courses. The primary endpoint, the GBS disability scale at 4 weeks, was assessed with multivariable ordinal regression.

Results of 237 eligible patients, 199 patients received a single IVIg course. Twenty patients received an 'early' second IVIg course (1-2 weeks after start of the first IVlg course) and 18 patients a 'late' second IVIg course (2-4 weeks after start of IVIg). At baseline and 1 week, those receiving two IVIg courses were more disabled than those receiving one course. Compared with the one course group, the adjusted OR for a better GBS disability score at 4 weeks was $0.70(95 \% \mathrm{Cl} 0.16$ to 3.04$)$ for the early group and $0.66(95 \% \mathrm{Cl} 0.18$ to 2.50$)$ for the late group. The secondary endpoints were not in favour of a second IVIg course.

Conclusions This observational study did not show better outcomes after a second IVIg course in GBS with poor prognosis. The study was limited by small numbers and baseline imbalances. Lack of improvement was likely an incentive to start a second IVIg course. A prospective randomised trial is needed to evaluate whether a second IVIg course improves outcome in GBS.

\section{INTRODUCTION}

A standard course of intravenous immunoglobulin (IVIg, $2 \mathrm{~g} / \mathrm{kg}$ in 2-5 days) shortens time to recovery in Guillain-Barré syndrome (GBS) when administered within the first 2 weeks. ${ }^{1-4}$ However, approximately $20 \%$ of patients are unable to walk independently at 6 months. ${ }^{5}$ Evidence-based treatment options to improve outcome are currently lacking. ${ }^{6}$ One small, uncontrolled series of 'treatment unresponsive' patients suggested that a second IVIg course was more effective than one course. In addition, patients with a large increment in serum IgG level after IVIg treatment recovered more quickly than those with a small increment. ${ }^{8}$ There are however reasons not to treat all GBS patients with a second IVIg course. First, approximately $80 \%$ of GBS patients treated with one IVIg course recover relatively well. ${ }^{5}$ Second, serious side effects may occur, including anaphylaxis, acute kidney injury, thromboembolic events or haemolytic anaemia. ${ }^{9} 10$ Third, IVIg is an expensive and relatively scarce blood product.

Therefore, careful selection of patients who might benefit from a second course of IVIg is important. The modified Erasmus GBS Outcome Score (mEGOS) identifies patients who are more likely to have a poor prognosis, defined as being unable to walk independently. ${ }^{11}$ These patients in particular might benefit from a second course of IVIg if administered within the first weeks after onset of disease, when nerve damage is most likely reversible. We used the database of the prospective, observational International Guillain-Barré Syndrome Outcome Study (IGOS) to compare disease course in patients treated with one IVIg course versus two IVIg courses and we aimed to assess whether a second IVIg course in patients with GBS and a predicted poor prognosis improved functional outcome. ${ }^{12}$

\section{METHODS}

Study design

IGOS is an ongoing, prospective, observational cohort study which includes patients with GBS within the first 2 weeks of onset. The IGOS study protocol has been published previously. ${ }^{12}$

\section{Study population and treatment}

For this International Second IVIg dose (ISID) study, we identified those treated with a standard course of $2 \mathrm{~g} / \mathrm{kg}$ IVIg over 2-5 consecutive days. As mEGOS has not been validated in young children, patients aged under 6 were excluded. ${ }^{11}$ We excluded patients who had died or were lost to follow-up in the first 7 days from study entry, or who received a second IVIg course because of a reported treatment related fluctuation (TRF) observed by the local physician. ${ }^{13}$ We also excluded patients who participated in a randomised controlled study (Second 
Immunoglobulin Dose in GBS patients (SID-GBS) trial ${ }^{14}{ }^{15}$ or Inhibition of Complement Activation in GBS (ICA-GBS) trial). ${ }^{16}$

Multiple imputation was used for patients with missing age $(n=9 / 1300)$ or medical research council (MRC) sum score at week $1(n=120 / 1300) .{ }^{17}$ Based on a standard set of five imputation samples, medians were calculated for age and MRC sum score. In this way, mEGOS could be calculated for all patients, using age, preceding diarrhoea and MRC sum score at week 1. We further identified patients with mEGOS 6-12 at 1 week who considered to have a poor prognosis $(35 \%$ probability or higher of not being able to walk independently at 6 months). ${ }^{11}$

\section{IVIg groups}

From the group of patients with poor prognosis treated with at least one IVIg course, we selected those treated with a second course of IVIg. Because of the observational nature of IGOS, the decision to administer two IVIg courses was made by the local treating investigators. As a result, the second IVIg course was not given at a standardised time point. In the analysis, we separated patients treated with a second IVIg course early (started within 2 weeks after start of the first IVIg course) from those treated late (started after 2 weeks but within 3-4 weeks after start of the first IVIg course and completed before the assessment of week 4). Patients who received one standard course of IVIg before the 4 week assessment were considered controls. Other additional treatments such as corticosteroids and plasma exchange (PE) were ignored.

\section{Assessments}

Demographic and clinical data including GBS disability score ${ }^{18}{ }^{19}$ MRC sum score,${ }^{20}$ sensory deficits, facial weakness, previous diarrhoea and clinical variants were collected at entry, and subsequently at week 1, 2 and 26 (GBS disability score, MRC sum score). ${ }^{12}$ According to the IGOS protocol, ${ }^{12}$ study entry should coincide with the first day of treatment, even if informed consent was obtained after start of treatment. Due to ethical regulations in some countries, study entry was set by the date of informed consent. Results of the first nerve conduction study were classified according to the criteria of Hadden and colleagues into demyelinating, axonal, inexcitable, equivocal or normal. ${ }^{21}$ Treatment information was collected regarding dates of start and end of treatment, treatment type (IVIg, PE, other), treatment regimen and side effects after IVIg.

Deterioration at the time of starting the second IVIg course was determined by worsening at least one MRC sum score point on the visits prior to and after the moment of starting the second IVIg course.

\section{Study endpoints}

The primary endpoint was improved functional outcome on the GBS disability scale after 4 weeks. Secondary endpoints were GBS disability score at 26 weeks, improvement of $\geq 1$ score on the GBS disability scale at 4 and 26 weeks, median change in the MRC sum score at 4 and 26 weeks, being able to walk independently at 26 weeks, requiring ventilation at any time during follow-up, time admitted to the intensive care unit (ICU), time on a ventilator, GBS related mortality at 6 months, TRF, and complications (not further specified).

\section{Statistical analysis}

Statistical analyses were performed using SPSS software V.21.0 and V.24.0. Data were expressed as medians with IQR or as proportions. Mann-Whitney $U$ tests were used to compare continuous variables across two groups, and one-way ANOVA or Kruskal-Wallis tests (if variances differed significantly) were used to compare continuous variables across three groups. $\chi^{2}$ or Fisher's exact tests were performed to compare proportions. Reported p-values were calculated between the three groups unless stated otherwise. A two-sided p-value $<0.05$ was considered to be significant. Treatment effect on the GBS disability scale at 4 and 26 weeks was evaluated for the early and late second IVIg groups using multivariable ordinal regression analysis, adjusting for prognostic factors and disease severity (age, GBS disability score at entry and week 1, MRC sum score at entry and week 1, occurrence of diarrhoea, electrophysiological axonal or inexcitable pattern) and country of residence. The reported OR express the odds of having a better outcome (ie, a lower GBS disability score).

\section{Sub-analysis: propensity score matching}

We recognised that the non-randomised study design could have caused confounding by indication due to observed and unobserved confounders. To correct for the effect of confounders, we developed a multivariable regression model and performed a propensity score matched analysis. With this method, propensity scores for receiving treatment were calculated for each individual, given an individual's covariates. ${ }^{22}$ We calculated the propensity scores for each individual in the multivariable logistic regression model with independent variables: age, gender, time to enter the study, time to start first IVIg course, GBS disability score at entry and week 1, MRC sum score at week 1, GBS variant at entry, preceding diarrhoea and country of residence. Variables with missing values would result in a lower number of matched controls and were therefore not added in the model (eg, electrophysiological classification, deterioration or improvement at starting the second course). In our model, the calculated propensity scores expressed the probability of receiving a second IVIg course. The propensity score was subsequently used to match controls to patients in both the early and late second IVIg group (nearest neighbour matching 1:1 with a calliper of 0.1 ). After propensity score matching, we performed a new unadjusted ordinal regression analysis.

\section{RESULTS}

\section{Patients}

In January 2017, 1300 patients with a follow-up period of 6 months had been enrolled in IGOS. Seventy-one patients $(5 \%)$ were excluded because of alternative diagnosis, $6(0.5 \%)$ because of protocol violation, 34 (3\%) because of young age and $29(2 \%)$ because of insufficient data.

Of the remaining 1165 patients, 831 (71\%) were initially treated with IVIg. Seventeen patients were lost to follow-up at the first week and seven died before 7 days after study entry, so that prognosis could be predicted in 807 patients based on the mEGOS. Poor prognosis (mEGOS 6-12) was predicted in 260 patients (32\%), of whom 23 were excluded because they participated in a randomised controlled trial (RCT, SID-GBS trial 11; ICA-GBS trial 1) or because they received the second IVIg course because of a TRF (11). Ultimately, 237 patients with a poor prognosis fulfilled the entry criteria for this study (figure 1).

\section{Control group}

The primary endpoint of this study concerned improvement at 4 weeks. Therefore, the control group included the 199 patients treated with one IVIg course within the first 4 weeks from study entry irrespective of other treatments before or after 4 weeks. Of 


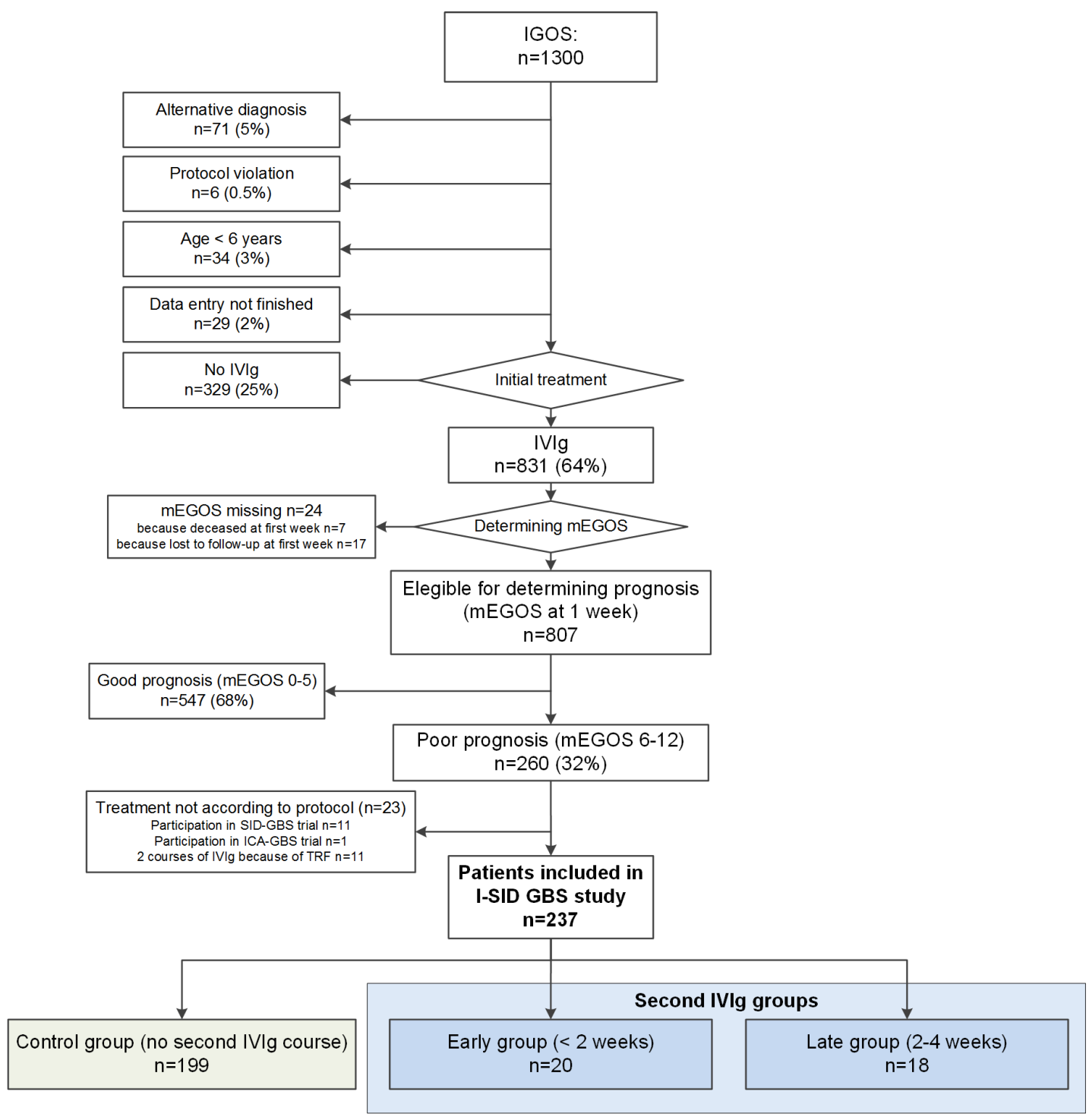

Figure 1 Patient selection for the I-SID study. GBS, Guillain-Barré Syndrome; ICA-GBS, Inhibition of Complement Activation in GBS; IGOS, International GBS Outcome Study; I-SID GBS, International Second Immunoglobulin Dose in GBS patients; IVIg, Intravenous immunoglobulin; mEGOS, modified Erasmus GBS Outcome Score; TRF, treatment related fluctuation.

the 199 patients, 160 (80\%) received standard treatment only (1 IVIg course within 4 weeks of study entry), 31 patients were additionally treated with PE before or after 4 weeks, and six patients were treated with additional IVIg after 4 weeks. One additional patient received IVIg within 4 weeks, followed by IVIg after 4 weeks and was thereafter treated because of vasculitis. Another patient received IVIg and 7 PE sessions within 4 weeks and was after his GBS diagnosed with granulomatous polyangiitis.

\section{Early second IVIg group}

Twenty patients were treated with a second IVIg course that started within 14 days after start of the first IVIg course and were included in the 'early second IVIg group'. Sixteen (80\%) were treated with only one additional IVIg course and four were treated with various combinations of IVIg and PE.

Late second IVIg group

Eighteen patients were treated with a second IVIg course two to 4 weeks after start of the first IVIg course and were included in the 'late second IVIg group'. Fourteen (78\%) were treated with only one additional IVIg course while four patients were treated with various combinations of IVIg and PE.

\section{Data completeness}

At week 4, the primary endpoint was available in 167/199 (84\%) patients in the control group (eight patients lost to follow-up and 24 missed the visit). In both the early and the late second IVIg group one patient was lost to follow-up and one patient had a missed visit. Therefore, the primary endpoint was available in $90 \%(18 / 20$ and $16 / 18)$ in the two IVIg groups.

\section{Patient characteristics}

At baseline, there were no significant differences between the three treatment groups regarding age, gender, MRC sum score at entry, sensory deficits, preceding diarrhoea or GBS variant (table 1). There were significant differences in the proportion of patients already ventilated at study entry in the early second IVIg group $(n=9,45 \%)$ and in the late second IVIg group $(n=6$, $33 \%)$ compared with the control group $(n=36,18 \%)$ (3-way $p$ value, $\mathrm{p}=0.01)$. 
Table 1 Demographic and clinical characteristics at entry and during disease course

\begin{tabular}{|c|c|c|c|c|}
\hline & $\begin{array}{l}\text { Control group } \\
(1 x \text { IVIg) } n=199\end{array}$ & $\begin{array}{l}\text { Early second IVIg group } \\
\text { ( } 2 x \text { IVIg) } n=20\end{array}$ & $\begin{array}{l}\text { Late second IVIg group } \\
(2 x I V I g) n=18\end{array}$ & $\begin{array}{l}\text { P value among three } \\
\text { groups* }\end{array}$ \\
\hline \multicolumn{5}{|l|}{ Demographics } \\
\hline Males, n (\%) & $109(55)$ & $12(60)$ & $12(67)$ & 0.58 \\
\hline Age, years, median (IQR) & $59(43-70)$ & $65(54-70)$ & $59(53-71)$ & 0.54 \\
\hline \multicolumn{5}{|l|}{ Clinical features at entry } \\
\hline Time from onset to study entry, days, median (IQR) & $5(3-8)$ & $4(2-8)$ & $5(2-8)$ & 0.68 \\
\hline Time from onset to first IVlg course, days, median (IQR) & $3(2-6)$ & $2(1-3)$ & $2(1-5)$ & 0.11 \\
\hline Antecedent diarrhoea, $\mathrm{n}(\%)$ & $73(37)$ & $4(20)$ & $5(28)$ & 0.27 \\
\hline Facial weakness, n (\%) & $71(36)$ & $8(40)$ & $9(50)$ & 0.47 \\
\hline MRC sum score, median (IQR) & $32(18-42)$ & $27(5-42)$ & $30(3-46)$ & 0.56 \\
\hline Sensory deficits, $n(\%)$ & $113(57)$ & $13(65)$ & $7(39)$ & 0.47 \\
\hline \multicolumn{5}{|l|}{ GBS variant, $\mathrm{n}(\%)$} \\
\hline No & $141(71)$ & $15(75)$ & $12(67)$ & 0.85 \\
\hline Pure motor & $46(23)$ & $3(15)$ & $6(33)$ & 0.41 \\
\hline Miller Fisher (overlap) & $10(5)$ & $2(10)$ & $0(0)$ & 0.37 \\
\hline Other & $2(1)$ & $0(0)$ & $0(0)$ & $\dagger$ \\
\hline \multicolumn{5}{|l|}{ Clinical features after 1 week follow-up } \\
\hline MRC sum score, median (IQR) & $25(8-35)$ & $10(0-26)$ & $6(1-32)$ & $0.004 \neq \S$ \\
\hline mEGOS, median (IQR) & $10(8-11)$ & $11(9-11)$ & $10(8-11)$ & 0.10 \\
\hline \multicolumn{5}{|l|}{ Clinical features at nadir } \\
\hline MRC sum score, median (IQR) & $21(4-33)$ & $4(0-20)$ & $2(0-16)$ & $<0.001 \neq \S$ \\
\hline \multicolumn{5}{|l|}{ GBS disability score, $n,(\%)$} \\
\hline Unable to run (2) & $1(1)$ & $0(0)$ & $0(0)$ & $0.03 \ddagger$ \\
\hline Unable to walk independently (3) & $7(4)$ & $0(0)$ & $0(0)$ & \\
\hline Bedridden or chairbound (4) & $107(54)$ & $4(20)$ & $6(33)$ & \\
\hline Ventilated (5) & $84(42)$ & $16(80)$ & $12(67)$ & \\
\hline \multicolumn{5}{|l|}{ Electrophysiological classification, n (\%) } \\
\hline Demyelinating & $87 / 154(57)$ & $9 / 12(75)$ & 9/17 (53) & $\dagger$ \\
\hline Axonal & 18/154 (12) & $1 / 12(8)$ & $5 / 17(29)$ & \\
\hline Inexcitable & $8 / 154(5)$ & $0(0)$ & $1 / 17(6)$ & \\
\hline Equivocal & 40/154 (26) & $2 / 12(17)$ & $2 / 17(12)$ & \\
\hline Normal & $1 / 154(1)$ & $0(0)$ & $0(0)$ & \\
\hline Time until NCS, days, median (IQR) & $6(4-10)$ & $2(2-10)$ & $7(4-9)$ & 0.52 \\
\hline
\end{tabular}

*There were no significant differences between the early and late second IVIg group.

tNot calculated because of small patient numbers.

$\ddagger P$-value $<0.05$ for control group versus early second IVIg group.

$\S \mathrm{P}$-value $<0.05$ for control group versus late second IVIg group.

GBS, Guillain-Barré syndrome; IVIg, intravenous immunoglobulin; mEGOS, modified Erasmus GBS Outcome Score; MRC, medical research council; NCS, nerve conduction study.

One week after study entry, patients in the early and late second IVIg group had significantly lower MRC sum scores (10, IQR 0-26, and 6, IQR 1-32) than controls (25, IQR 8-35) $(p=0.004)$ and were thus more severely affected. This was also reflected by higher GBS disability scores (figure 2).

Patients in the control group were often already improving at least one point on the MRC sum score between the first to the second study week $(n=102,63 \%)$. However, patients in the second IVIg groups were often still deteriorating at least one point in MRC sum score at the time of starting their second IVIg course $(n=13,81 \%$ in the early group and $n=8,47 \%$ in the late group).

\section{Primary endpoint}

Treatment with a second IVIg course made no significant difference to the GBS disability score 4 weeks after study entry. The adjusted OR for a lower GBS disability score was 0.70 (95\% CI 0.16 to 3.04$)$ for the early second IVIg group, and 0.66 (95\% CI 0.18 to 2.50 ) for the late group (figure 2 , table 2).

\section{Secondary endpoints}

There was also no significant difference in the GBS disability score at 26 weeks. The adjusted OR for a lower GBS disability score was 0.89 for the early group $(95 \% \mathrm{CI} 0.22$ to 3.53$)$ and 0.40 (95\% CI 0.10 to 1.62) for the late group (figure 2, table 2).

Fifty-one $(31 \%)$ patients in the control group improved at least one point on the GBS disability scale 4 weeks after study entry, compared with only $3(17 \%, p=0.22)$ of the early group and none $(p=0.01)$ of the late group (table 3$)$. At 26 weeks, 127 of $145(88 \%)$ patients in the control group improved at least one point on the GBS disability scale compared with $12 / 16(75 \%$, $p=0.16)$ in the early and only $4 / 11(36 \%, p<0.001)$ in the late IVIg group (table 3).

In the control group, patients improved by a median of 4 points on the MRC sum score (IQR -8 to 12 ) from entry to 4 weeks. The MRC sum score decreased by two points (IQR -23 to 10$)$ in the early group $(\mathrm{p}=0.14)$ and by four points (IQR -36 to 2$)$ in the late group $(\mathrm{p}<0.001)$. After 26 weeks, the patients in the early second IVIg group improved more on the MRC sum 


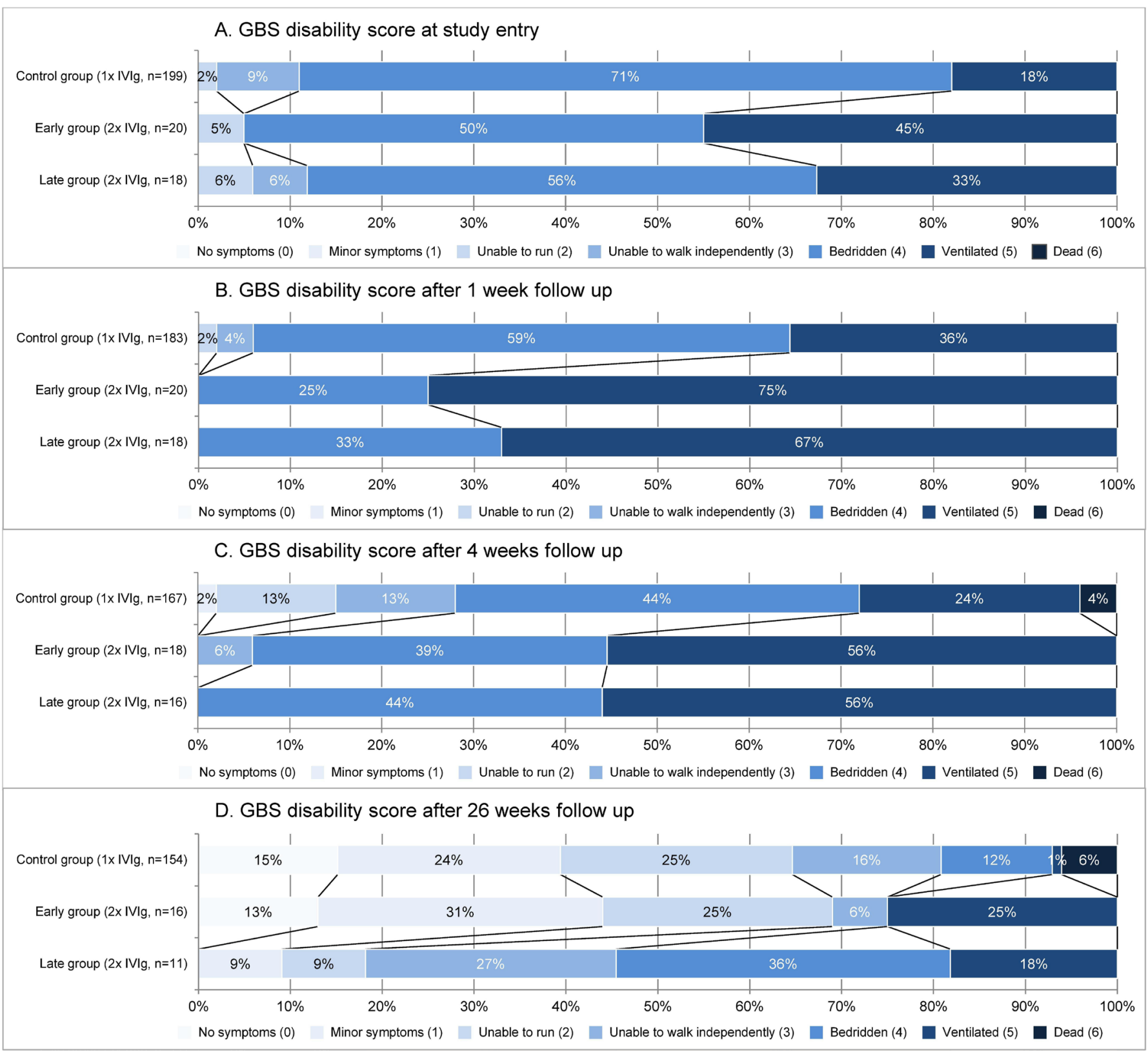

Figure 2 GBS disability score at study entry (A), 1 (B), 4 (C) and 26 weeks (D). GBS, Guillain-Barré syndrome; IVIg, intravenous immunoglobulin.

\begin{tabular}{|c|c|c|c|c|c|c|}
\hline & $\mathrm{N}$ & $\begin{array}{l}\text { Unadjusted OR } \\
(95 \% \mathrm{Cl})\end{array}$ & $P$ value & $\mathrm{N}$ & $\begin{array}{l}\text { Adjusted OR* } \\
(95 \% \mathrm{Cl})\end{array}$ & $P$ value \\
\hline \multicolumn{7}{|l|}{ Week 4: } \\
\hline \multicolumn{7}{|l|}{ Treatment } \\
\hline Control & 167 & Ref. & & 125 & Ref. & \\
\hline Early second IVIg & 18 & 0.31 (0.12 to 0.78$)$ & 0.01 & 10 & 0.70 (0.16 to 3.04 ) & 0.63 \\
\hline Late second IVlg & 16 & 0.28 (0.11 to 0.76$)$ & 0.01 & 14 & 0.66 (0.18 to 2.50$)$ & 0.54 \\
\hline \multicolumn{7}{|l|}{ Week 26: } \\
\hline \multicolumn{7}{|l|}{ Treatment } \\
\hline Control & 154 & Ref. & & 105 & Ref. & \\
\hline Early second IVIg & 16 & 0.98 (0.39 to 2.44$)$ & 0.97 & 8 & 0.89 (0.22 to 3.53$)$ & 0.87 \\
\hline Late second IVIg & 11 & 0.23 (0.08 to 0.70$)$ & 0.01 & 8 & 0.40 (0.10 to 1.62$)$ & 0.40 \\
\hline
\end{tabular}

\footnotetext{
${ }^{*}$ Adjusted for age, preceding diarrhoea, GBS disability score at entry and week 1, MRC sum score at entry and week 1, axonal or inexcitable NCS, country of residence.
} GBS, Guillain-Barré syndrome; IVIg, intravenous immunoglobulin; MRC, medical research council; NCS, nerve conduction study. 
Table 3 Endpoints at 4 and 26 weeks

\begin{tabular}{|c|c|c|c|c|}
\hline & $\begin{array}{l}\text { Control group } \\
(1 x \text { IVlg) } n=199\end{array}$ & $\begin{array}{l}\text { Early second IVlg group } \\
(2 x \text { IVIg) } n=20\end{array}$ & $\begin{array}{l}\text { Late second IVIg group } \\
(2 x \text { IVlg) } n=18\end{array}$ & $P$ value \\
\hline \multicolumn{5}{|l|}{ Secondary endpoints } \\
\hline \multicolumn{5}{|c|}{ Improving $\geq 1$ score on GBS disability sore, $\mathrm{n}(\%)$ at: } \\
\hline 4 weeks & $51 / 167(31)$ & $3 / 18(17)$ & $0(0)$ & $0.002^{*} \dagger$ \\
\hline 26 weeks & 127/145 (88) & $12 / 16(75)$ & $4 / 11(36)$ & $0.001 * \dagger$ \\
\hline \multicolumn{5}{|l|}{ Able to walk independently, $n(\%)$ at: } \\
\hline 26 weeks & $99 / 154(64)$ & $11 / 16(69)$ & $2 / 11(18)$ & $0.01^{*} \dagger \ddagger$ \\
\hline \multicolumn{5}{|l|}{ Change in MRC sum score (median, IQR) at: } \\
\hline 4 weeks & $4(-8$ to 12$)$ & $-2(-23$ to 10$)$ & $-4(-36$ to 2$)$ & $<0.001$ \\
\hline 26 weeks & $18(12-32)$ & $27(3-48)$ & $11(-4$ to 21$)$ & 0.06 \\
\hline Requiring ventilation, $\mathrm{n}(\%)$ & $88(44)$ & $16(80)$ & $12(67)$ & $0.003 \S$ \\
\hline GBS related mortality at 6 months, $n(\%)$ & 9/154 (6) & $0(0)$ & $0(0)$ & 0.44 \\
\hline TRFף, n (\%) & $4(2)$ & $2(10)$ & $1(6)$ & 0.11 \\
\hline \multicolumn{5}{|l|}{ Complications after first IVlg course, $\mathrm{n}(\%)$} \\
\hline Headache & $3(2)$ & $0(0)$ & $1(6)$ & \\
\hline Shivering & $1(1)$ & $0(0)$ & $0(0)$ & \\
\hline Nausea/vomiting & $1(1)$ & $0(0)$ & $0(0)$ & \\
\hline Hallucinations/psychosis & $0(0)$ & $1(5)$ & $0(0)$ & \\
\hline Hypo/hypertension & $1(1)$ & $0(0)$ & $0(0)$ & \\
\hline \multicolumn{5}{|l|}{ Complications after second IVlg course, $\mathrm{n}(\%)$} \\
\hline Headache & $0(0)$ & $0(0)$ & $1(6)$ & \\
\hline
\end{tabular}

*P value derived from unadjusted binary logistic regression analysis.

tP value $<0.05$ for control group versus late second IVIg group.

$\ddagger P$ value $<0.05$ for early versus late second IVIg group.

$\S P$ value $<0.05$ for control group versus early second IVIg group.

१The second IVIg course in the early and late groups was not given because of the TRF.

GBS, Guillain-Barré syndrome; IVIg, intravenous immunoglobulin; MRC, medical research council; TRF, treatment related fluctuation.

score (median change 27, IQR 3-48) than the control group (18, IQR $12-32, \mathrm{p}=0.43)$ and the late group $(11, \mathrm{IQR}-4$ to 21 , $\mathrm{p}=0.05)$.

Treatment related fluctuations were reported, but not treated with a second IVIg course, in 4 of the control patients, 2 of the early group patients and in 1 patient of the late group.

ICU admission was longest in patients after late treatment (64 days, IQR 33-144), whereas the controls (30 days, IQR 13-55) and patients in the early group (31 days, IQR 18-82) had similar ICU admission stays. Patients in the late group required longer ventilatory support (76 days, IQR 33-239) than the controls (27 days, IQR 15-61) and early group (55 days, IQR 26-220).

Serious complications of the second IVIg courses were not reported. Six control patients experienced headache, shivering, nausea or vomiting and/or blood pressure changes after their first IVIg course. In the early group, one patient had hallucinations/psychosis and in the late group, one patient experienced headache after the first IVIg course. Headache was reported in one patient after the second full IVIg course.

Nine patients in the control group died within 6 months $(6 \%)$ while in the second IVIg groups, no patients died. Causes of death were: cardiac arrest as a consequence of multi-organ system failure $(n=2)$, respiratory failure $(n=2$, of whom one chose to have ventilator support withdrawn after 2 weeks), pneumonia and sepsis $(n=2)$ and other $(n=3)$.

\section{Sub-analysis: ordinal regression analysis after propensity score matching}

Patients from the early and late second IVIg group were matched separately to controls by propensity scores. The unadjusted OR for a lower GBS disability score was calculated for the early and late group separately. The highest OR for a lower GBS disability score was found at 26 weeks for the early group $(1.26,95 \% \mathrm{CI}$ 0.35 to 4.60 ) but this was not statistically significant. The other ORs were also not in favour of a second IVIg course (table 4).

\section{DISCUSSION}

This is the first prospective study evaluating outcome after a second course of IVIg in patients with GBS. We did not observe a benefit from a second course of IVIg in GBS patients with a poor prognosis as defined by the mEGOS prognostic model. Severe complications such as haemolytic anaemia or thromboembolism were not reported after the second course of IVIg.

Table 4 ORs for a lower GBS disability score at 4 and 26 weeks after propensity score matching.

\begin{tabular}{|c|c|c|c|}
\hline & $\mathbf{N}$ & $\begin{array}{l}\text { Unadjusted OR } \\
(95 \% \mathrm{Cl})\end{array}$ & $P$ value \\
\hline \multicolumn{4}{|l|}{ Week 4: } \\
\hline \multicolumn{4}{|l|}{ Treatment } \\
\hline Control & 18 & Ref. & \\
\hline Early second IVIg & 18 & 0.74 (0.21 to 2.64$)$ & 0.64 \\
\hline Control & 16 & Ref. & \\
\hline Late second IVIg & 16 & $1.03(0.26$ to 4.13$)$ & 0.97 \\
\hline \multicolumn{4}{|l|}{ Week 26: } \\
\hline \multicolumn{4}{|l|}{ Treatment } \\
\hline Control & 16 & Ref. & \\
\hline Early second IVIg & 16 & $1.26(0.35$ to 4.60$)$ & 0.73 \\
\hline Control & 12 & Ref. & \\
\hline Late second IVIg & 11 & $0.42(0.09$ to 1.90$)$ & 0.26 \\
\hline
\end{tabular}


After 1 week, patients in the second IVIg groups were significantly more disabled (lower MRC sum scores and higher GBS disability scores) than the IVIg controls. These patients also were deteriorating more often at the start of their second IVIg course compared with the one IVIg course group (deteriorating at least one point on the MRC sum score: $13 / 16,81 \%$ in the early group, $8 / 17,47 \%$ in the late group). Conversely, patients treated with one IVIg course were often already improving when a second course was administered in the second IVIg groups (ie, 102/163, $63 \%$ improving from the first to the second IGOS study week, and $112 / 151,74 \%$ improving from the second to the fourth IGOS study week). Continued deterioration was therefore the most likely reason for the treating physicians to start a second IVIg course, whereas improvement probably prevented starting a second course. The unbalanced disease severity likely caused confounding by indication because a poor neurological condition may have influenced the investigators' decision to initiate a second IVIg treatment but likely also resulted in a worse outcome. Despite correcting for disease severity in a multivariable ordinal regression model, the data did not show a beneficial effect from a second course of IVIg.

Duration of ICU stay and ventilation were secondary endpoints. These situations however also may have prompted the decision to administer a second course of IVIg; a higher proportion of patients who received a second IVIg course had longer ICU admission and required assisted ventilation. The median time on a ventilator was longer than the time admitted to the ICU in all groups because in some countries patients were discharged to rehabilitation centres with mechanical ventilation facilities.

In addition to observed confounding factors, unbalanced unobserved confounders likely played a role too. This is demonstrated by the difference between unadjusted and adjusted ORs. One of the unobserved confounders could be $\operatorname{IgG}$ or albumin levels. ${ }^{83}$ In this study we did not have data on IgG and serum albumin concentrations. Other unobserved confounders could have been insurance status, availability of IVIg, and other unknown patient, physician or hospital related factors. In our attempt to mitigate the effect of confounders, we conducted a secondary analysis in which we matched patients on propensity scores, defined as the probability of receiving an early or late second IVIg course. Even with this analysis, the data did not show positive ORs for a better outcome.

In order to prevent further nerve damage, treatment might be most effective in the early stage of GBS. In our study, only eight patients received a second IVIg course within 9 days after start of the first IVIg course, while the other patients received the second IVIg course later, possibly because they were in a poor neurological condition. Furthermore, 20\% of the patients in all three IVIg groups were also treated with PE, or with more than two IVIg courses or combinations of PE and IVIg. Approximating the preferred analysis of a randomised controlled trial, we conducted an 'intention-to-treat' analysis. Therefore, we did not exclude patients treated with IVIg combined with other treatments and also not the two patients in the control group who later on were diagnosed with vasculitis and granulomatous polyangiitis. A large RCT showed that IVIg administered immediately after PE was not better than IVIg or PE alone. ${ }^{1}$ No randomised trials have been performed to evaluate the effect of PE after IVIg. However, since PE removes IVIg, this sequence of treatment should logically be avoided, or used only at least 2 weeks after IVIg. By that stage however any treatment is likely to have only marginal effects as nerve damage has already occurred. ${ }^{6}$
We selected patients with a poor prognosis because we expected that these patients might benefit most from a second IVIg course. These patients have previously been identified to have a probability of $35 \%$ or greater of not being able to walk independently. ${ }^{11}$ This does not mean that all patients with a high mEGOS score have poor outcome. The predictive value of the mEGOS has been validated recently in another cohort of 177 patients where a significant correlation was found between higher mEGOS and poor outcomes. ${ }^{24}$

Next to the major limitation of the observational nature of this study, other limitations can be pointed out. First, the 4 week endpoint was chosen as the primary outcome because it corresponds to the primary endpoint in previous randomised controlled trials. However, it may be that this time point was not best suited for this observational study, especially in the late group, since the GBS disability score was usually recorded less than 1 week after the completion of the second IVIg course. Second, in ordinal regression analysis the treatment effect ideally should be the same across all cut-off values of the outcome scale (the proportional odds assumption), but in this study the treatment effect was not similar across the GBS disability scale (figure 2). However, it has been argued in the statistical literature that the proportional odds model is still valid when the proportional odds assumption is not met. ${ }^{25}$ Lastly, despite starting with a large group of GBS patients and using multiple imputation to increase the number of eligible subjects, we ultimately had small numbers in the second IVIg groups ( $\mathrm{n}=20$ in the early group and $\mathrm{n}=18$ in the late group).

In conclusion, the observational design of this large prospective multicentre international study introduced bias by observed and unobserved confounding factors. This study however reflects current daily practice in GBS patients with a poor prognosis, and showed no positive effect of a second IVIg course on functional outcome. The second IVIg course was often started late, and this was likely because of severe neurological impairment after a standard IVIg course. A positive effect of a second IVIg course cannot be ruled out but needs to be investigated further as is being done in the SID-GBS RCT. ${ }^{14} 15$

\section{Author affiliations}

${ }^{1}$ Department of Neurology, Erasmus MC, Rotterdam, The Netherlands ${ }^{2}$ Department of Neurology, Johns Hopkins University, Baltimore, Maryland, USA ${ }^{3}$ Department of Public Health, Erasmus MC, Rotterdam, The Netherlands ${ }^{4}$ Department of Neurology, St. Elizabeth's Medical Center, Boston, Massachusetts, USA

${ }^{5}$ Department of Neurology, National Hospital for Neurology and Neurosurgery, London, UK

${ }^{6}$ Department of Neurology, University Clinic St. Luc, Leuven, Belgium

${ }^{7}$ Department of Neurology, Aarhus University Hospital, Aarhus, Denmark

${ }^{8}$ Department of Neurology, University of Cape Town, Cape Town, South Africa

${ }^{9}$ Department of Clinical Neurophysiology, Reference Centre for NMD, Nantes

University Hospital, Nantes, France

${ }^{10}$ Department of Neurology, Odense University Hospital, Odense, Denmark

${ }^{11}$ Department of Neurology, Kindai University Faculty of Medicine, Osaka, Japan

${ }^{12}$ Department of Neurology, Royal Victoria Infirmary, Newcastle, UK

${ }^{13}$ Department of Laboratory Sciences and Services Division, The International Centre for Diarrhoeal Disease Research, Dhaka, Bangladesh

${ }^{14}$ Department of Neurology, Heinrich Heine University, Düsseldorf, Germany

${ }^{15}$ Department of Neurology, University of Glasgow, Glasgow, UK

${ }^{16}$ Department of Immunology, Erasmus MC, Rotterdam, The Netherlands

${ }^{17}$ MRC Centre for Neuromuscular Diseases, National Hospital for Neurology and

Neurosurgery, London, UK

Correction notice This article has been corrected since it was published Online First. Table data have been aligned correctly and footnotes adjusted.

Twitter Zhahirul Islam @na

Acknowledgements We thank the patients who participated in this long term follow up study. 
Collaborators For this study, patients were selected from the following collaborators: J.M. Addington, MD (University of Virginia, Charlottesville, USA); S Ajroud-Driss, MD (Northwestern University Feinberg, Chicago, USA); G. Antonini, MD (Mental Health and Sensory Organs (NESMOS), Sapienza University, Sant'Andrea Hospital, Rome, Italy); S. Attarian, MD, PhD (CHU Timone, Marseille, France); F.A. Barroso, MD (Instituto de Investigaciones Neurológicas Raúl Carrea, FLENI, Buenos Aires, Argentina); L. Benedetti, MD, PhD (Ospedale Sant' Andrea La Spezia, La Spezia, Italy); T.E. Bertorini, MD (The University of Tennessee Health Science Center (UTHSC), Memphis, USA); T.H. Brannagan, MD (Columbia University, New York City, USA); C. Briani, MD (University of Padova, Padova, Italy); R. Bhavaraju-Sanka, MD (University Hospital/University of Texas Health Science Center, San Antonio Texas, USA); S. Butterworth, MD (Pinderfields Hospital, Wakefield, UK); C. Casasnovas, MD, PhD (Bellvitge University Hospital - IDIBELL Neurometabolic Diseases Group. CIBERER, Barcelona, Spain); G. Cavaletti, MD (University Milano-Bicocca, Monza, Italy); S. Chen, MD, PhD (Rutgers, Robert Wood Johnson University Hospital, New Brunswick, USA); K.G. Claeys, MD, PhD (1. University Hospitals Leuven, Leuven, Belgium, 2. KU Leuven, Leuven, Belgium); J.S. Cosgrove, MD (Leeds General Infirmary, Leeds, UK); A Davidson, MD (University of Glasgow, Glasgow, UK); E. Dardiotis, MD (University of Thessaly, Hospital of Larissa, Larissa, Greece); C. Dornonville de la Cour, MD (National Hospital Copenhagen, Copenhagen, Denmark); C.G. Faber, MD, PhD (Maastricht University Medical Centre, Maastricht, The Netherlands); T.E. Feasby, MD (University of Calgary, Calgary, Canada); T. Fujioka, MD (Toho University Medical Center, Tokyo, Japan); G. Galassi, MD (University Hospital of Modena, Modena, Italy); J.M. Gilchrist, MD (Soulthern Illinois University School of Medicine, Springfield, USA); N. A. Goyal, MD (University of California, Irvine, USA); V. Granit, MD (Montefiore Medical, Center, New York, USA); G. Gutiérrez-Gutiérrez, MD (Hospital Universitario Infanta Sofia, San Sebastian, Spain); R.D.M. Hadden, MD, PhD (King's College Hospital, London, UK); J.K.L. Holt, PhD, FRCP (The Walton Centre, Liverpool, UK); M. Htut, MD (St. George's Hospital, London, UK); I. Jericó Pascual, MD, PhD (Complejo Hospitalario de Navarra, Pamplona, Spain); S. Karafiath, MD (University of Utah School of Medicine, Salt Lake City, USA); H.D. Katzberg, MD (University of Toronto, Toronto, Canada); L. Kiers, MD (University of Melbourne, Royal Melbourne Hospital, Parkville, Australia); B.C. Kieseier, MD (Heinrich Heine University, Düsseldorf, Germany); K. Kimpinski, MD (University Hospital, LHSC, London-Ontario, Canada); S. Kuwabara, MD, PhD (Chiba University, Chiba, Japan); J.Y. Kwan, MD (University of Maryland School of Medicine, Baltimore, USA); S.S. Ladha, MD (Barrow Neurology Clinics, Phoenix, Arizona, USA); V. Lawson, MD (Wexner Medical Center at The Ohio State University, Columbus, USA); H. Lehmann, MD, PhD (University Hospital of Cologne, Universitätsklinikum Köln, Cologne, Germany); H. Manji, MD, FRCP (Ipswich Hospital, Ipswich, UK); G.A. Marfia, MD (Neurological Clinic, Policlinico Tor Vergata, Rome, Italy); C. Márquez Infante, MD (Hospital Universitario Virgen del Rocio, Seville, Spain); M.G. Mattiazzi, MD (Hospital Militar Central, Buenos Aires, Argentina); C.J. McDermott, MD (Royal Hallamshire Hospital, NIHR Clinical, Sheffield, UK); M.S. Monges, MD (Hospital de Pediatría J.P. Garrahan, Buenos Aires, Argentina); G. Morís de la Tassa, MD (Hospital Universitario Central de Asturias, Asturias, Spain); C. Nascimbene, MD, PhD (Luigi Sacco Hospital, Milan, Italy); E. Nobile Orazio, MD, PhD (Milan University, Humanitas Clinicala and Research Institute Milan, Italy); R.J. Nowak, MD (Yale University School of Medicine, New Haven, USA); M. Osei-Bonsu (James Cook University Hospital, Middlesbrough, UK); J. Pardo Fernandez (Hospital Clínico de Santiago, Santiago de Compostela (A Coruña), Spain); L. Querol Gutierrez, MD, PhD (Hospital de la Santa Creu i Sant Pau, Universitat Autònoma de Barcelona, Barcelona, Spain); R. Reisin (Hospital Britanico, Buenos Aires, Argentina); S. Rinaldi, $\mathrm{MBChB}, \mathrm{PhD}$ (University of Oxford R.C. Roberts, MD (Addenbrooke's Hospital Cambridge, Cambridge, UK); I. Rojas-Marcos, MD (Hospital Univesitario Reina Sofia, Cordoba, Spain); S.A. Rudnicki, MD (University of Arkansas, Fayetteville, USA); A. Schenone, MD, PhD (1. Department of Neurosciences, Rehabilitation, Ophthalmology, Genetics and Maternal and Infantile Sciences (DINOGMI), University of Genova, Genova, Italy 2. IRCCS Policlinico San Martino, Genova, Italy); M.J. Sedano Tous, MD (Hospital Universitario Marques de Valdecilla, Santander, Cantabria, Spain); N. Shahrizaila, MD (Neurology Unit, Department of Medicine, Faculty of Medicine, University of Malaya, Malaya); K. Sheikh, MD, PhD (The University of Texas Health Science Center at Houston, Houston, USA); N.J. Silvestri, MD (Buffalo General Medical Center, Buffalo NY, USA); C.L. Sommer, MD (Universitätsklinikum Würzburg, Würzburg, Germany); J.D. Varrato, DO (Lehigh Valley Health Network, Allentown, USA); J. Verschuuren, MD, PhD (Leiden University Medical Centre, Leiden, The Netherlands); M.V. Vytopil, MD, PhD (Tufts University School of Medicine Lahey Hospital W. Waheed, MD (University of Vermont Medical Center, Burlington, USA); L. Zhou, MD, PhD (Icahn School of Medicine at Mount Sinai, New York, USA).Other collaborators were:U.A. Badrising, MD, PhD (Leiden University Medical Centre, Leiden, The Netherlands); I.R. Bella, MD (University of Mass Medical School, Worcester, USA); C. Bunschoten, MD, PhD candidate (Erasmus University Medical Centre, Rotterdam, The Netherlands); J. Bürmann, MD, Universitätsklinikum des Saarlandes, Homburg, Germany); M. Busby, MD (Leeds General Infirmary, Bradford, UK); C.C. Chao, MD, PhD (National Taiwan University Hospital, Taipei, Taiwan); M.E. Conti, MD (University Hospital Clinicas, Buenos Aires, Argentina); M.C. Dalakas, MD (1. Thomas Jefferson University, Philadelphia, USA, 2. National and Kapodistrian University of Athens, Athens, Greece); P. Van Damme, MD, PhD (University Hospital Leuven, Leuven, Belgium); A. Doets, MD, PhD candidate (Erasmus University Medical
Centre, Rotterdam, the Netherlands); G.W. van Dijk, MD (Canisius Wilhelmina Hospital, Nijmegen, The Netherlands); M.M. Dimachkie, MD (University of Kansas Medical Center, Kansas City, USA); K. Doppler, MD (Universitätsklinikum Würzburg, Würzburg, Germany); A. Echaniz-Laguna, MD (Hopital de Hautepierre, Strasbourgh, France); F. Eftimov, MD, PhD (Amsterdam University Medical Centre, Amsterdam, The Netherlands); R. Fazio, MD (Scientific Institute San Raffaele, Milan, Italy); C. Fokke, MD (Gelre Hospital, Zutphen and Apeldoorn, The Netherlands); E.A. Fulgenzi, MD (Hospital Cesar Milstein Buenos Aires, Buenos Aires, Argentina); M.P.J. Garssen, MD, PhD (Jeroen Bosch Hospital, 's Hertogenbosch, Zaltbommel and Drunen, The Netherlands); C.J. Gijsbers, MD (Vlietland Hospital, Schiedam, The Netherlands); J. Gilhuis, MD, PhD (Reinier de Graaf Gasthuis, Delft, The Netherlands); A. Grapperon, MD (CHU Timone, Marseille, France); S.T. Hsieh, MD, PhD (National Taiwan University Hospital, Taipei, Taiwan); I. Illa, MD, PhD (Hospital de la Santa Creu i Sant Pau, Universitat Autònoma de Barcelona, Barcelona, Spain); B. Islam, MD, PhD (International Centre for Diarrhoeal Disease Research, Bangladesh (icddr,b), Dhaka, Bangladesh); K. Jellema, MD, PhD (Haaglanden Medisch Centrum, The Hague, The Netherlands); K. Kaida, MD, PhD (National Defense Medical College, Saitama, Japan); N. Kokubun, MD (Dokkyo Medical University, Tochigi, Japan); N. Kolb, MD (University of Vermont, Burlington VT, USA); R. van Koningsveld, MD, PhD (Elkerliek Hospital, Helmond and Deurne, The Netherlands); A.J. van der Kooi, MD, PhD (Amsterdam University Medical Centre, Amsterdam, The Netherlands); K. Kuitwaard, MD, PhD (Albert Schweitzer Hospital, Dordrecht, The Netherlands); L. Landschoff Lassen, MD (Glostrup Hospital, Glostrup, Denmark); S.E. Leonhard, MD, PhD candidate (Erasmus University Medical Centre, Rotterdam, the Netherlands); M. Mandarakas, MD, PhD (Erasmus University Medical Centre, Rotterdam, the Netherlands); E. Martinez Hernandez, MD (Institut d'Investigacions Biomèdiques August Pi i Sunyer (IDIBAPS), Hospital Clinic, Barcelona, Spain); Q.D. Mohammad, MD, PhD (National Institute of Neurosciences and Hospital, Dhaka, Bangladesh); M. Pulley, MD (University of Florida, Jacksonville, USA); Y.A. Rajabally, MD, PhD (Queen Elizabeth Hospital, Birmingham, UK); S.W. Reddel, MD, PhD (Concord Repatriation General Hospital, Sydney, Australia); T. van der Ree, MD (Westfriesgasthuis, Hoorn, The Netherlands); J. Roodbol, MD, PhD candidate (Erasmus University Medical Centre, Rotterdam, the Netherlands); G.M. Sachs, MD (University of Rhode Island, Providence, USA); J.P.A. Samijn, MD, PhD (Maasstad Hospital, Rotterdam, The Netherlands); L. Santoro, MD, PhD (University Federico II, Napels, Italy); B. Stein, MD (St. Joseph's Regional Medical Center, Paterson, USA); F.H. Vermeij, MD (Franciscus Gasthuis, Rotterdam, The Netherlands); L.H. Visser, MD, PhD (Elisabeth-TweeSteden Hospital, Tilburg and Waalwijk, The Netherlands); H.J. Willison, MD, PhD (University of Glasgow, Glasgow, UK); P. Wirtz, MD, PhD (HagaZiekenhuis, The Hague, The Netherlands); S.A. Zivkovich, MD, PhD (University of Pittsburgh Medical Center, Pittsburgh, USA).

Contributors CV: Study concept and design, patient inclusion, acquisition of data, analysis and interpretation of the data, drafted and revised the manuscript for intellectual content. Member of IGOS Coordinating Center, IGOS Country Coordinator. BvdB: Study concept and design, patient inclusion, acquisition of data, interpretation of the data, revised the manuscript for intellectual content. Member of IGOS Coordinating Center, IGOS Country Coordinator. DRC: Study concept and design, interpretation of the data, revised the manuscript for intellectual content. Member of the IGOS Steering Committee. EV and $\mathrm{HL}$ : Analysis and interpretation of the data, revised the manuscript for intellectual content. KCG: Study concept and design, interpretation of the data, revised the manuscript for intellectual content. Member of the IGOS Steering Committee, IGOS Country Coordinator. MPL: Study concept and design, patient inclusion, acquisition of data, interpretation of the data, revised the manuscript for intellectual content. PVdB, TH, KB, YP, SK, ZI, GC: Patient inclusion, acquisition of data, interpretation of the data, revised the manuscript for intellectual content. IGOS Country Coordinator. SHS, JM: Patient inclusion, acquisition of data, interpretation of the data, revised the manuscript for intellectual content. $\mathrm{H}-\mathrm{PH}$ : Study concept and design, interpretation of the data, revised the manuscript for intellectual content. Member of the IGOS Steering Committee. BCJ: Study concept and design, patient inclusion, acquisition of data, interpretation of the data, revised the manuscript for intellectual content. Member of the IGOS Steering Committee, Member of the IGOS Coordinating Center, IGOS Country coordinator. RH: Study concept and design, interpretation of the data, revised the manuscript for intellectual content. Member of the IGOS Steering Committee. PvD: Study concept and design, patient inclusion, acquisition of data, analysis and interpretation of the data, drafted and revised the manuscript for intellectual content. Member of the IGOS Steering Committee.

Funding This study is mainly funded by Grifols. Other sponsors of IGOS are GBSCIDP Foundation International, GAIN Charity, Erasmus University Medical Centre, Glasgow University, CSL Behring and Annexon.

Competing interests $\mathrm{CV}, \mathrm{BvdB}, \mathrm{EV}, \mathrm{HL}, \mathrm{KB}, \mathrm{SHS}, \mathrm{ZI}$ report no disclosures. DRC is a consultant for Acetylon, Alcobra Pharma, Alnylam Pharmaceuticals, Annexon Biosciences, Akros Pharma, Biotest Pharmaceuticals, Boehringer Ingelheim, Cigna HealthManagement, CSL Behring, DP Clinical, Grifols, Hansa Medical, Karos Pharmaceuticals, Neurocrine Biosciences, Novartis, Octapharma, Pharnext, Seattle Genetics, Sun Pharmaceuticals, and Syntimmune. He is on the data and safety monitoring board for Sanofi, Pledpharma, Pfizer, Johnson Roche, Sanofi Genzyme, 
Teva, TG Therapeutics and UCB. GC has received honoraria from CSL Behring. BCJ has received funding for research projects from Prinses Beatrix Spierfonds, Horizon 2020,GBS-CIDP Foundation International, Grifols, CSL Behring and Annexon. He is on the Medical Advisory Board for the GBS-CIDP Foundation International, and a member of the Inflammatory Neuropathy Consortium. RH has current consultancies with LFB and former consultancies with Novartis. PvD has received honoraria for consulting, lectures and serving on steering committees from Octapharma, Kedrion, CSL Behring, Grifols, and Hansa (all honoraria to departmental research fund), and is currently receiving grants from Prinses Beatrix Spierfonds, Sanquin Blood supply, Shire and Grifols. He is President Elect of the Peripheral Nerve Society, member of the Inflammatory Neuropathy Consortium, Medical Advisory Board for the GBS-CIDP Foundation International, editorial board for the Journal of the Neurological Sciences and Journal of Neuromuscular Diseases. He is PI of the RCT investigating the effect of methylprednisolone in GBS (MP/IVIg RCT in GBS) and the RCT investigating the effect of a second dose IVIg in GBS (SID-GBS study).

Patient consent for publication Not required.

Provenance and peer review Not commissioned; externally peer reviewed.

Data availability statement Data are available upon reasonable request.

\section{ORCID iDs}

Christine Verboon http://orcid.org/0000-0003-1086-6679

Susumu Kusunoki http://orcid.org/0000-0002-1584-8704

\section{REFERENCES}

1 Randomised trial of plasma exchange, intravenous immunoglobulin, and combined treatments in Guillain-Barré syndrome. plasma Exchange/Sandoglobulin Guillain-Barré syndrome trial group. Lancet 1997;349:225-30.

2 Hughes RAC, Swan AV, Raphaël J-C, et al. Immunotherapy for Guillain-Barré syndrome: a systematic review. Brain 2007;130:2245-57.

3 Hughes RAC, Swan AV, van Doorn PA, et al. Intravenous immunoglobulin for GuillainBarré syndrome. Cochrane Database Syst Rev 2014;46. CD002063.

4 van der Meché FG, Schmitz PI. A randomized trial comparing intravenous immune globulin and plasma exchange in Guillain-Barré syndrome. Dutch Guillain-Barré Study Group. N Engl J Med 1992;326:1123-9.

5 van den Berg B, Walgaard C, Drenthen J, et al. Guillain-Barré syndrome: pathogenesis, diagnosis, treatment and prognosis. Nat Rev Neurol 2014;10:469-82.

6 Verboon C, van Doorn PA, Jacobs BC. Treatment dilemmas in Guillain-Barré syndrome. J Neurol Neurosurg Psychiatry 2017;88:346-52.

7 Farcas P, Avnun L, Frisher S, et al. Efficacy of repeated intravenous immunoglobulin in severe unresponsive Guillain-Barré syndrome. Lancet 1997;350:1747.

8 Kuitwaard K, de Gelder J, Tio-Gillen AP, et al. Pharmacokinetics of intravenous immunoglobulin and outcome in Guillain-Barré syndrome. Ann Neurol 2009;66:597-603.
9 Ammann EM, Haskins CB, Fillman KM, et al. Intravenous immune globulin and thromboembolic adverse events: a systematic review and meta-analysis of RCTs. Am J Hematol 2016;91:594-605.

10 Nguyen TP, Biliciler S, Wahed A, et al. Occurrence of hemolytic anemia in patients with GBS treated with high-dose IVIg: table. Neurol Neuroimmunol Neuroinflamm 2014; $1:$ :50

11 Walgaard C, Lingsma HF, Ruts L, et al. Early recognition of poor prognosis in GuillainBarre syndrome. Neurology 2011;76:968-75.

12 Jacobs BC, van den Berg B, Verboon C, et al. International Guillain-Barré syndrome outcome study: protocol of a prospective observational cohort study on clinical and biological predictors of disease course and outcome in Guillain-Barré syndrome. J Peripher Nerv Syst 2017;22:68-76.

13 Ruts L, Drenthen J, Jacobs BC, et al. Distinguishing acute-onset CIDP from fluctuating Guillain-Barre syndrome: a prospective study. Neurology 2010;74:1680-6.

14 SID-GBS trial. Available: http://www.trialregister.nl/trialreg/admin/rctview.asp?TC= 2224

15 Walgaard C, Jacobs BC, Lingsma HF, et al. Second IVIg course in Guillain-Barré syndrome patients with poor prognosis (SID-GBS trial): protocol for a double-blind randomized, placebo-controlled clinical trial. J Peripher Nerv Syst 2018;23:210-5.

16 Davidson Al, Halstead SK, Goodfellow JA, et al. Inhibition of complement in GuillainBarré syndrome: the ICA-GBS study. J Peripher Nerv Syst 2017;22:4-12.

17 Donders ART, van der Heijden GJMG, Stijnen T, et al. Review: a gentle introduction to imputation of missing values. J Clin Epidemiol 2006;59:1087-91.

18 Hughes RAC, Newsom-Davis JM, Perkin GD, et al. Controlled trial of prednisolone in acute polyneuropathy. The Lancet 1978;312:750-3.

19 Fokke C, van den Berg B, Drenthen J, et al. Diagnosis of Guillain-Barré syndrome and validation of Brighton criteria. Brain 2014;137:33-43.

20 Kleyweg RP, van der Meché FG, Schmitz PI. Interobserver agreement in the assessment of muscle strength and functional abilities in Guillain-Barré syndrome. Muscle Nerve 1991;14:1103-9.

21 Hadden RD, Cornblath DR, Hughes RA, et al. Electrophysiological classification of Guillain-Barré syndrome: clinical associations and outcome. plasma Exchange/ Sandoglobulin Guillain-Barré syndrome trial group. Ann Neurol 1998;44:780-8.

22 Klungel $\mathrm{OH}$, Martens EP, Psaty BM, et al. Methods to assess intended effects of drug treatment in observational studies are reviewed. J Clin Epidemiol 2004:57:1223-31.

23 Fokkink W-JR, Walgaard C, Kuitwaard K, et al. Association of albumin levels with outcome in intravenous Immunoglobulin-Treated Guillain-Barré syndrome. JAMA Neurol 2017;74:189-96.

24 Yamagishi Y, Suzuki H, Sonoo M, et al. Markers for Guillain-Barré syndrome with poor prognosis: a multi-center study. J Peripher Nerv Syst 2017;22:433-9.

25 Senn S, Julious S. Measurement in clinical trials: a neglected issue for statisticians? Stat Med 2009;28:3189-209. 